Fourth International Conference on Sustainable Construction Materials and Technologies http://www.claisse.info/Proceedings.htm

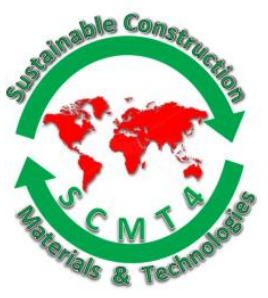

SCMT4

Las Vegas, USA, August 7-11, 2016

\title{
Long-Term Infrastructure Durability Enhancement of Hybrid Fiber-Reinforced Concrete Under Corrosive Environments
}

\author{
Wilson Nguyen ${ }^{1 \mathrm{a}}$, Rotana Hay ${ }^{1 \mathrm{~b}}$, Gabriel Jen ${ }^{1 \mathrm{c}}$, and Claudia P. Ostertag ${ }^{1 \mathrm{~d}}$ \\ ${ }^{1}$ Department of Civil \& Environmental Engineering, University of California, Berkeley, CA, USA.

 \\ ${ }^{1 c}$ Email<gjen@berkeley.edu>, ${ }^{1 d}$ Email <ostertag@ce.berkeley.edu>.
}

\begin{abstract}
With the eventual replacement of deteriorated reinforced concrete structures to occur in the United States for the next several decades, the use of novel construction materials should be considered to promote structures with enhanced durability and service life. In this study, the primary strategy for resisting damage caused by corrosive environments is through the multi-scale crack control provided by hybrid fiberreinforced concrete (HyFRC). To reduce the consumption of virgin materials in concrete construction and better incorporate recycled byproducts, two additional variants of the HyFRC composite design are investigated - a green HyFRC (G-HyFRC) utilizing 50\% fly ash inclusion (by binder mass) and recycled concrete aggregate, as well as a high-performance green HyFRC (HP-G-HyFRC) utilizing $60 \%$ fly ash and slag inclusion. Because reinforced concrete structures are subjected to various crack-inducing loads while in service, the influence of cracking prior to corrosion activity was accounted for to determine its impact on the resulting permeability of HyFRC and G-HyFRC composites and conventional concrete. Current results reveal the crack resistance provided by fiber hybridization during mechanical loading, including suppression of splitting cracks, resulted in a considerable increase in corrosion initiation time compared to conventional concrete. Induced corrosion of reinforced HP-G-HyFRC by impressed current methods revealed less damage compared to reinforced concrete specimens without fiber reinforcement.
\end{abstract}

\section{INTRODUCTION}

Reinforced concrete is a composite material that is susceptible to various forms of deterioration while in service. Recent evaluations on the condition of the United States' infrastructure revealed approximately $11 \%$ of the nation's 607,000 bridges were classified as structurally deficient, requiring rehabilitation or complete replacement due to possible reduction in load-carrying capacity (American Society of Civil Engineers, 2013). In particular, the corrosion of steel is the costliest issue considering all durability-related phenomena. Past corrosion-related repair and maintenance costs have been set to 8.3 billion dollars annually for all infrastructure (Koch et al., 2002). Considering structurally deficient bridges account for approximately 33\% of total decking area in the United States (American Society of Civil Engineers, 2013), indicating the structures requiring the most attention for repair or replacement are large in scale, the volume of consumed materials for such construction is significant. Because portland cement is produced at a rate 
of 3.6 billion tonnes annually (U.S. Geological Survey, 2013) and is subsequently responsible for approximately $7 \%$ of global anthropogenic carbon dioxide emissions (IEA-WBCSD, 2009), premature rehabilitation or replacement of corroded reinforced concrete structures prior to designed service life completion is undesirable from a carbon dioxide emissions standpoint.

To improve the durability of reinforced concrete against corrosion damage, use of fiber reinforcement for matrix crack control may be effective. The short, discontinuous fibers bridge crack faces, requiring increased stress for additional opening and propagation compared to conventional concrete. When the maximum concrete crack width remains under a critical threshold value, found by others to be up to 0.1 to $0.2 \mathrm{~mm}$, the diffusion of deleterious external solutions is comparable to uncracked concrete (Wang et al., 1997, Sahmaran and Yaman, 2008). Such damage tolerance leads to an increase in corrosion initiation time. Because the formation of internal expansive corrosion products in the propagation phase cracks the surrounding concrete matrix, an increase in the crack resistance of the concrete is thought to mechanically suppress the growth of such products. Several types of fiber-reinforced concrete have shown to be effective in mitigating corrosion-induced damage (Sahmaran et al., 2008, Miyazato and Hiraishi, 2013, Berrocal et al., 2015).

The research presented herein investigates the feasibility of improving the sustainability of reinforced concrete primarily by mechanical crack control provided by fiber reinforcement. Hybrid fiber-reinforced concrete (HyFRC) is selected for study. This concrete uses multiple types of fibers to achieve multi-scale crack control from initial microcracking to macrocrack propagation (Blunt and Ostertag, 2009). To further reduce the embodied energy of HyFRC composites, additional HyFRC variants with high recycled byproduct substitution are considered for performance. HyFRC and green HyFRC (G-HyFRC), detailed with $50 \%$ cement mass replacement with fly ash and complete replacement of virgin coarse aggregate with recycled concrete aggregate, were subjected to flexural preloading followed by long-term saltwater intrusion. A high-performance green HyFRC (HP-G-HyFRC), detailed with $60 \%$ cement replacement with fly ash and slag, was investigated under accelerated corrosion methods to determine the crack resistance enhancement provided by hybrid fiber reinforcement. In all cases, HyFRC composites were found to significantly increase resistance against corrosion-induced damage compared to conventional concrete.

\section{EXPERIMENTAL PROGRAM}

\section{Materials}

Mix proportions of the three types of HyFRC variants evaluated for corrosion performance are presented in Table 1. Additionally, control specimens consisting of concrete (C), green concrete (G-C), and highperformance green concrete (HP-G-C) were studied. Binding materials consisted of a combination of type I/II portland cement (PC), class F fly ash (FA), and blast-furnace slag. G-HyFRC and G-C were proportioned with $50 \%$ replacement of portland cement mass with fly ash. Due to the high-volume inclusion of fly ash, the water-binder ratio of the green concrete was reduced from the typical HyFRC design (0.54) to increase early-age strength, with a value of 0.40 deemed acceptable. HP-G-HyFRC achieved a high 28day compressive strength of $86 \mathrm{MPa}$ despite $15 \%$ and $45 \%$ binder proportion of fly ash and slag, respectively. The water-binder ratio was set to 0.25 to further improve strength properties compared to HyFRC. The development of HP-G-HyFRC is further detailed by Hay and Ostertag (2014).

For all concrete types in the study, fine aggregates consisted of sand with a fineness modulus of 3.2. Coarse aggregates were included in all mix designs, consisting of either pea gravel with a maximum size of 9.5 $\mathrm{mm}$ or recycled concrete aggregate (RCA). The RCA was obtained from a construction materials recycling center located in Oakland, California, located approximately $22 \mathrm{~km}$ from the University of California, 
Berkeley campus (Figure 1a). The as-received product contained phases of mortar and larger aggregate from the original demolished structure, with the RCA having a maximum size of $9.5 \mathrm{~mm}$. For proper use in concrete batching, particles less than $4.8 \mathrm{~mm}$ in size were removed and the remaining material was washed (Figure 1b, 1c).

Table 1. Concrete mix proportions $\left(1 \mathrm{~m}^{3}\right.$ of concrete).

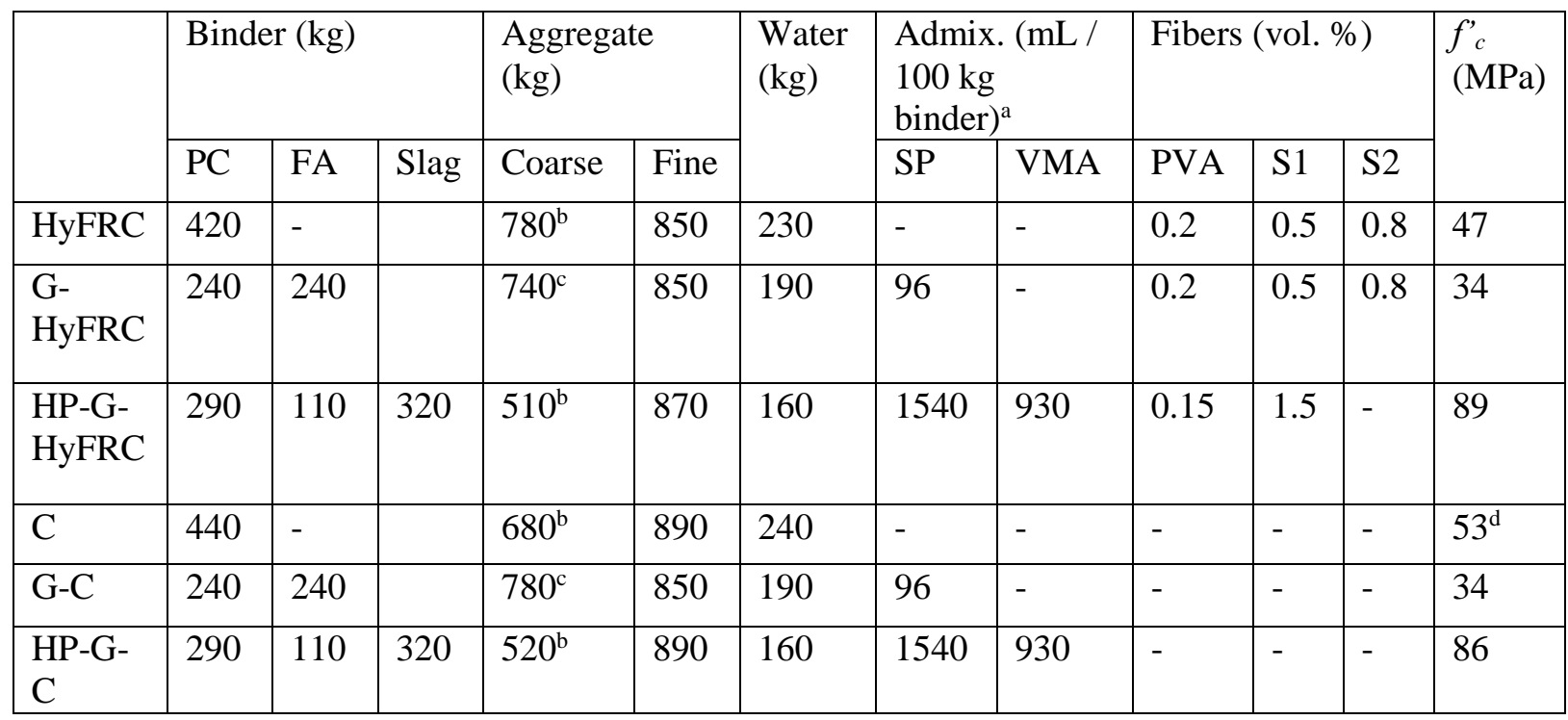

$a-S P$ and VMA refer to superplasticizer and viscosity-modifying admixture, respectively.

$b$ - Pea gravel; $c$-Recycled concrete aggregate.

$d$-Specimens not subjected to preload were cast in a separate batch, with $f^{\prime}{ }_{c}=45 \mathrm{MPa}$.

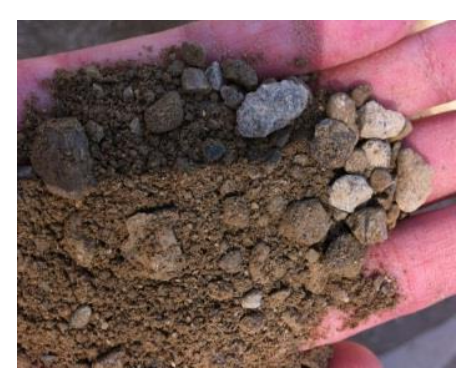

(a)



(b)



(c)

Figure 1. Recycled concrete aggregate: (a) As-received at recycling center; (b) Sieved and washed; (c) Final product for casting, shown with scale reference.

For all concrete types in the study, fine aggregates consisted of sand with a fineness modulus of 3.2. Coarse aggregates were included in all mix designs, consisting of either pea gravel with a maximum size of 9.5 $\mathrm{mm}$ or recycled concrete aggregate (RCA). The RCA was obtained from a construction materials recycling center located in Oakland, California, located approximately $22 \mathrm{~km}$ from the University of California, Berkeley campus (Figure 1a). The as-received product contained phases of mortar and larger aggregate from the original demolished structure, with the RCA having a maximum size of $9.5 \mathrm{~mm}$. For proper use in concrete batching, particles less than $4.8 \mathrm{~mm}$ in size were removed and the remaining material was washed (Figure 1b, 1c). 
Table 2. Fiber properties.

\begin{tabular}{|l|l|l|l|l|l|l|}
\hline & Material & $\begin{array}{l}\text { Length } \\
(\mathrm{mm})\end{array}$ & $\begin{array}{l}\text { Diameter } \\
(\mathrm{mm})\end{array}$ & $\begin{array}{l}\text { Aspect ratio } \\
(\text { Length/Diameter } \\
)\end{array}$ & $\begin{array}{l}\text { Strength } \\
(\mathrm{MPa})\end{array}$ & $\begin{array}{l}\text { Elastic } \\
\text { modulus (GPa) }\end{array}$ \\
\hline PVA & PVA & 8 & 0.04 & 200 & 1600 & 43 \\
\hline S1 & Hooked-end steel & 30 & 0.55 & 55 & 1100 & 200 \\
\hline S2 & Hooked-end steel & 60 & 0.75 & 80 & 1050 & 200 \\
\hline
\end{tabular}

Fiber reinforcement for the HyFRC and G-HyFRC composites followed the same design, consisting of 0.2 volume percent of PVA fibers, $0.5 \%$ of $30 \mathrm{~mm}$-long steel fibers, and $0.8 \%$ of $60 \mathrm{~mm}$-long steel fibers. Properties of the fibers are provided in Table 2. The fiber proportioning was sufficient to develop deflectionhardening behavior in flexural elements. Due to workability issues with fresh HP-G-HyFRC using the same fiber reinforcement scheme, the PVA fiber volume fraction was reduced to $0.15 \%$ and $60 \mathrm{~mm}$-long steel fibers were removed. To compensate, the fiber volume fraction of $30 \mathrm{~mm}$-long steel fibers was increased to $1.5 \%$. The resulting composite achieved desired deflection-hardening behavior.

\section{Beam specimen design and preparation}

Beam specimens with dimensions of $152 \mathrm{~mm}$ by $152 \mathrm{~mm}$ by $610 \mathrm{~mm}$ were flexurally preloaded to induce cracking prior to long-term saltwater penetration (Figure 2a). The beams were cast with HyFRC, G-HyFRC, C, or G-C. A No. 3 (9.5 mm diameter) and a No. 6 (19 mm diameter) A615 steel reinforcing bar were embedded longitudinally along the beam length, with the cover between the rebar to the nearest horizontal concrete surface being $25 \mathrm{~mm}$. Prior to casting, the bars were sandblasted to remove the mill scale, such that variable corrosion effects caused by mill scale cracking would not be present (Ghods et al., 2011). A central length of $508 \mathrm{~mm}$ of both bars was allowed to bond directly to concrete and corrode under the experimental conditions. Surfaces of the rebar outside this region of interest were treated to prevent corrosion, first by coating with an electrically-insulating lacquer, followed by coating with electrical tape, and finished with a heat-shrunk polyolefin tubing. The two bars in each specimen were connected with an electrical wire to establish a galvanic cell. Because rebar in actual structures are in direct contact with other rebar in the concrete and corrode at varied times, the effects of macrocell corrosion were taken into account in the beam design.

The specimens were subjected to four-point flexural loading with a beam span of $457 \mathrm{~mm}$ and equidistant lengths between loading points $(152 \mathrm{~mm})$. Loading occurred such that concrete near the No. 3 rebar and No. 6 rebar was placed in tension and compression, respectively. After completion of loading, a polyvinyl chloride ponding dam was fixed to the horizontal surface of the beam nearest the No. 3 rebar (Figure 2b). The ponding dam allowed for continuous ingress of $3.5 \%$ sodium chloride solution (by mass) into the concrete matrix. Finally, a waterproofing sealant was applied to the surfaces of the specimens, except for the surface enclosed by the ponding dam footprint, and the entire horizontal surface opposite the ponding dam and nearest the No. 6 rebar. This surface established a directional flow of sodium chloride solution towards an evaporation surface during ponding. 




(a)



(b)

Figure 2. Beam design: (a) Detailing (mm); (b) Finished specimen.

HyFRC and plain concrete specimens were ponded continuously for the initial 11 weeks of experimentation, after which the specimens were subjected to alternating cycles of one week of ponding and one week of drying (complete relief of dam). The drying cycle was to allow sufficient oxygen to reach the rebar after penetrating the concrete cover. Specimens were stored in ambient laboratory conditions during the entirety of the induced corrosion portion of the experiment. Green composites were exposed to differing conditions. To expedite the corrosion chemical reactions, G-HyFRC and G-C specimens were stored in an environmental chamber with conditions of $50{ }^{\circ} \mathrm{C}$ and $50 \%$ relative humidity. After 82 weeks, corrosion measurements suggested all initially uncracked specimens remained in a passive state. At that time, the specimens were removed from the environmental chamber and placed in ambient laboratory conditions. It was expected that oxygen could more easily permeate through the concrete and increase corrosion activity.

\section{Cylinder specimen design and preparation}

Due to the reduced permeability of the high-performance concrete matrix and its high-strength properties, chloride penetration through uncracked sections of HP-G-HyFRC and HP-G-C was expected to require a corrosion initiation time in excess of practical experimental duration. To determine the long-term corrosion damage resistance of these materials, the corrosion initiation period was bypassed by adding solid sodium chloride pellets directly into the batching water during concrete specimen fabrication, resulting in dispersed chlorides throughout the matrix. The sodium chloride mass was proportioned at 4 percent of binder mass.

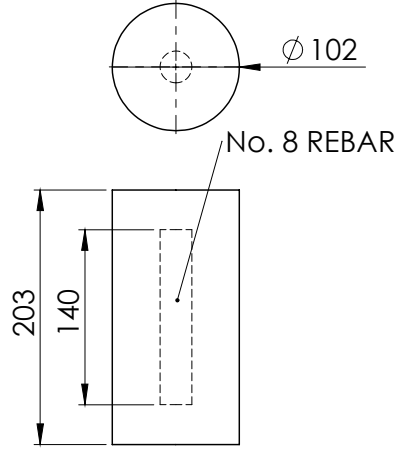

(a)



(b)

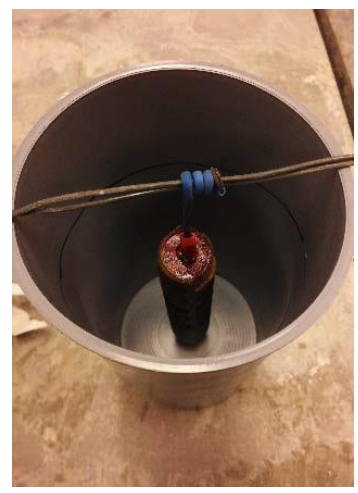

(c)

\section{Figure 3. Cylinder design: (a) Detailing (mm); (b) Sandblasted rebar; (c) Casting setup.}

Because the corrosion propagation performance was of interest, beam specimens were not required as the effect of chloride ingress through the depth of the concrete cover was not necessary. Cylindrical specimens, 
as shown in Figure 3a, were selected instead to study the type of radial cracking that would be caused by hoop stresses derived from internal corrosion product expansion. The gross concrete dimensions were set to $102 \mathrm{~mm}$ diameter by $203 \mathrm{~mm}$ height, and a $140 \mathrm{~mm}$-long No. 8 ( $25 \mathrm{~mm}$ diameter) rebar was centrally embedded along the cylinder's longitudinal axis. The bar was sandblasted and end surfaces of the bar were coated with an electrically-insulating lacquer (Figure 3b). During casting, the rebar was hung such that it was entirely embedded in the concrete, as shown in Figure 3c. An electrical wire was attached to the top end of the bar, and was allowed to extrude from the concrete.

HP-G-HyFRC and HP-G-C cylinder specimens underwent a differing corrosion testing protocol than the beams due to emphasis on corrosion propagation-related cracking. As previously mentioned, the concrete was cast with sodium chloride in the batching water to bypass the corrosion initiation stage. To further expedite results, these specimens were subjected to an impressed current of $112 \mathrm{~mA}$, based on a current density of $1 \mathrm{~mA} / \mathrm{cm}^{2}$, for either 24 or 48 continuous hours. The testing durations correlate to 2.7 and 5.5 years of non-accelerated testing, assuming all of the applied current participates in iron oxidation, based on the corrosion studies of reinforced HyFRC by Blunt (2008). Samples were immersed in a $17.5 \%$ sodium chloride solution during testing. After completion of accelerated testing, specimens were allowed to bathe in sodium chloride solution for 14 days while additional electrochemical measurements were taken.

\section{RESULTS AND DISCUSSION}

\section{Mechanical behavior of beams}

Beam specimens prescribed for flexural preloading were subjected to a set load level in five consecutive loading cycles. Figure 4 shows the load-displacement response of all preloaded beams. Specimens are designated by material and peak load (in $\mathrm{kN}$ ), though are not individually labeled. Green composite (GHyFRC and G-C) specimens were loaded to $31 \mathrm{kN}$. The load level was selected as being sufficient to induce observable flexural and splitting (longitudinal) cracks in the G-C specimens. No observable cracking was found in G-HyFRC specimens, which had an elastic response during loading.

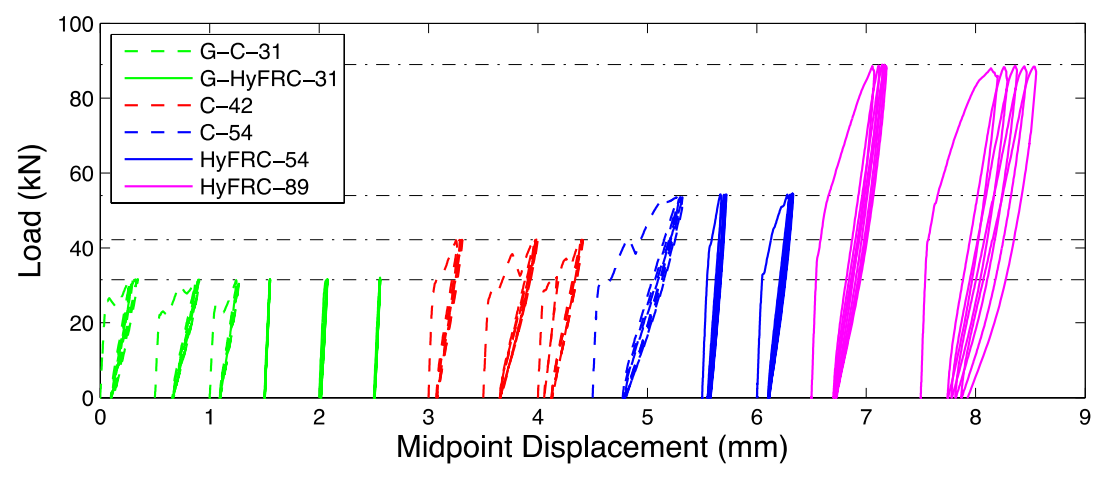

Figure 4. Load-displacement response of preloaded beams. Responses are offset for clarity.

To evaluate the effect of varied cracking states on beams, including the influence of cracks on HyFRC materials, samples cast with plain concrete and HyFRC were subjected to a greater range of peak load levels. Plain concrete specimens reached peak loads of $42 \mathrm{kN}$ or $54 \mathrm{kN}$. This range caused visible flexural cracking in all beams, with most specimens exhibiting splitting cracks. In comparison, HyFRC specimens reached peak loads of $54 \mathrm{kN}$ or $89 \mathrm{kN}$, as loading to $42 \mathrm{kN}$ did not produce any visible flexural cracks in the HyFRC composites. The $89 \mathrm{kN}$ load case represented an extreme scenario, as the load magnitude was $85 \%$ of the ultimate value found for monotonically loaded, failed beams. 
Table 3. Damage characteristics of preloaded beams, averaged per set.

\begin{tabular}{|l|l|l|l|l|l|l|}
\hline & $\begin{array}{l}\text { Max. disp. } \\
(\mathrm{mm})\end{array}$ & $\begin{array}{l}\text { Residual } \\
\text { disp. }(\mathrm{mm})\end{array}$ & $\begin{array}{l}\text { No. flexural } \\
\text { cracks }\end{array}$ & $\begin{array}{l}\text { Max. flexural } \\
\text { crack width } \\
(\mathrm{mm})\end{array}$ & $\begin{array}{l}\text { Split. crack } \\
\text { length } \\
(\mathrm{mm})\end{array}$ & $\begin{array}{l}\text { Split. crack } \\
\text { width (mm) }\end{array}$ \\
\hline G-C-31 & 0.34 & 0.13 & 2 & 0.13 & 84 & 0.04 \\
\hline $\begin{array}{l}\text { G-HyFRC- } \\
31\end{array}$ & 0.06 & 0.01 & - & - & - & - \\
\hline C-42f & & 0.36 & 0.11 & 2 & & 0.19 \\
\hline C-42s
\end{tabular}

$a-C-42 f$ specimens exhibited only observable flexural cracks.

$b-C-42 s$ specimens exhibited observable flexural and splitting cracks.

A summary of the specimens' damage characteristics is provided in Table 3. Individual specimen details are averaged within a set based on concrete type and peak load. The effectiveness of hybrid fiber reinforcement is realized when comparing the surface crack dimensions. While preloaded G-C-31 samples had an average maximum flexural crack width of $0.13 \mathrm{~mm}$ and had observable splitting crack formation, counterpart G-HyFRC-31 samples showed no surface cracking despite being subjected to the same cyclic loading protocol. After resisting peak loads of $42 \mathrm{kN}$, plain concrete (C) specimens exhibited average maximum flexural crack widths in the range of $0.17 \mathrm{~mm}$ to $0.19 \mathrm{~mm}$, while $54 \mathrm{kN}$ of peak load resulted in significantly smaller crack widths $(0.04 \mathrm{~mm})$ in HyFRC specimens despite greater applied loads. HyFRC specimens showed no surface splitting cracks up to $89 \mathrm{kN}$ of load, while plain concrete specimens showed such cracks beginning at $42 \mathrm{kN}$.

\section{Corrosion behavior of HyFRC beams}

Figure 5 plots the microcell corrosion current density $\left(i_{\text {corr }}\right)$ of the No. 3 rebar for plain concrete and HyFRC specimens over a duration of 52 weeks. The values were calculated assuming Stern-Geary coefficients based on measured corrosion potentials, and carry a maximum error factor of 2 using this method (Andrade and Alonso, 1996). Negligible corrosion is assumed for $i_{\text {corr }}$ values less than $0.1 \mu \mathrm{A} / \mathrm{cm}^{2}$. Active corrosion may be possible for greater values, however due to the error factor, active corrosion is likely for $i_{\text {corr }}$ measurements greater than $0.2 \mu \mathrm{A} / \mathrm{cm}^{2}$. Within the initial 10 weeks of monitoring, measurements suggested passive behavior in all specimens, excluding the results of HyFRC-89, which exhibited noticeably higher corrosion activity during the same period despite not forming splitting cracks and not having the greatest maximum flexural crack width among sample sets. While the behavior was not expected, internal cracking in HyFRC-89 specimens may have caused the early corrosion initiation. 


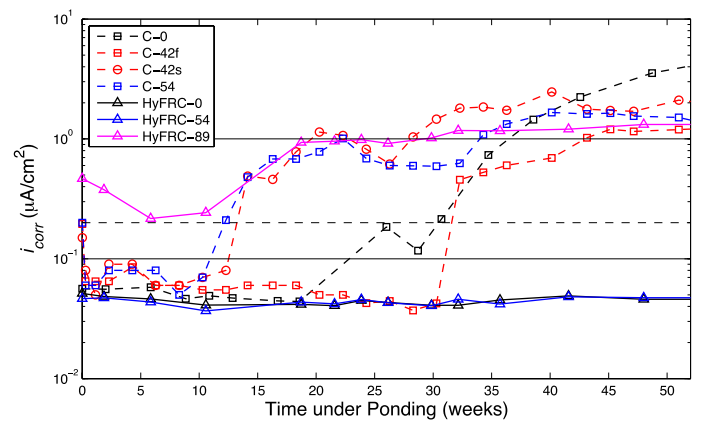

Figure 5. Microcell corrosion current density of No. 3 rebar in C and HyFRC beams.

The presence of splitting cracks was found to have a significant influence on the corrosion initiation time of specimens. Measurements for C-42s and C-54, which were the only specimens among C and HyFRC samples that formed splitting cracks as a result of preloading, sharply increased between the $12^{\text {th }}$ and $14^{\text {th }}$ week of experimentation, surpassing the active threshold value of $0.2 \mu \mathrm{A} / \mathrm{cm}^{2}$. After this time, the current density of C-42s was generally greater than that of C-54, which may be attributed to the longer splitting crack width of C-42s (191 mm versus $114 \mathrm{~mm}$ ) providing expedited chloride migration to a greater surface area of the rebar. Because splitting cracks are formed at the rebar-matrix interface, where the deformed ribs of the rebar bear on the concrete and produce hoop stresses around the rebar (Goto, 1971), cracks observed on the surface of concrete specimens penetrate to the depth of rebar embedment, providing chloride solution a direct path to the rebar. Remaining plain concrete specimens ( $\mathrm{C}-0$ and $\mathrm{C}-42 \mathrm{f}$ ) reached the active corrosion threshold after 31 to 33 weeks. The C-0 specimens, despite not having cracks as a result of preloading, may have achieved a similar corrosion initiation time as the $\mathrm{C}-42 \mathrm{f}$ specimens due to a higher matrix permeability, as suggested by its lower measured compressive strength (45 MPa versus $53 \mathrm{MPa}$ ). When comparing the corrosion initiation times of the C-42f and C-42s specimens, C-42f likely exhibited a delay in activity due to the absence of splitting cracks reaching the surface of the concrete specimen, as the maximum flexural crack width between C-42f and C-42s were similar.

HyFRC-54 specimens showed no significant increase in current density during the considered 52 weeks. Due to only minor surface cracking in these specimens $(0.04 \mathrm{~mm}$ crack width), chloride penetration through the concrete matrix was similar to uncracked HyFRC specimens (HyFRC-0). The result is consistent with the critical crack width for water permeability influence being in the range of up to 0.1 to $0.2 \mathrm{~mm}$, as previously discussed (Wang et al., 1997, Sahmaran and Yaman, 2008). By limiting crack growth, HyFRC composites can behave similarly to uncracked concrete while still resisting service loads that would otherwise cause flexural cracks greater than 0.10 to $0.20 \mathrm{~mm}$ and splitting cracks in reinforced plain concrete, as was the case for specimen C-54.

\section{Corrosion behavior of G-HyFRC beams}

Measurements of the microcell corrosion current density for G-C and G-HyFRC beams are presented in Figure 6 for 104 weeks of experimentation. Unlike the $\mathrm{C}$ and HyFRC specimens, $i_{\text {corr }}$ in these samples was based on Tafel scan tests that directly provided Stern-Geary coefficients, resulting in measurement values without an inherent error factor. Using the lower bound of assumed corrosion activity, G-C-31 specimens may have been actively corroding as early as 14 weeks under ponding, based on $i_{\text {corr }}$ exceeding $0.1 \mu \mathrm{A} / \mathrm{cm}^{2}$. Because these specimens exhibited surface splitting cracks, it is not surprising that the corrosion initiation time was similar to C-42s and C-54, which also had such cracks. For the presented two years of testing, the average corrosion current density of the other specimens (G-C-0, G-HyFRC-0, and G-HyFRC-31) generally remained under $0.1 \mu \mathrm{A} / \mathrm{cm}^{2}$, while G-C-31 reached a peak $i_{\text {corr }}$ of $7.3 \mu \mathrm{A} / \mathrm{cm}^{2}$. 


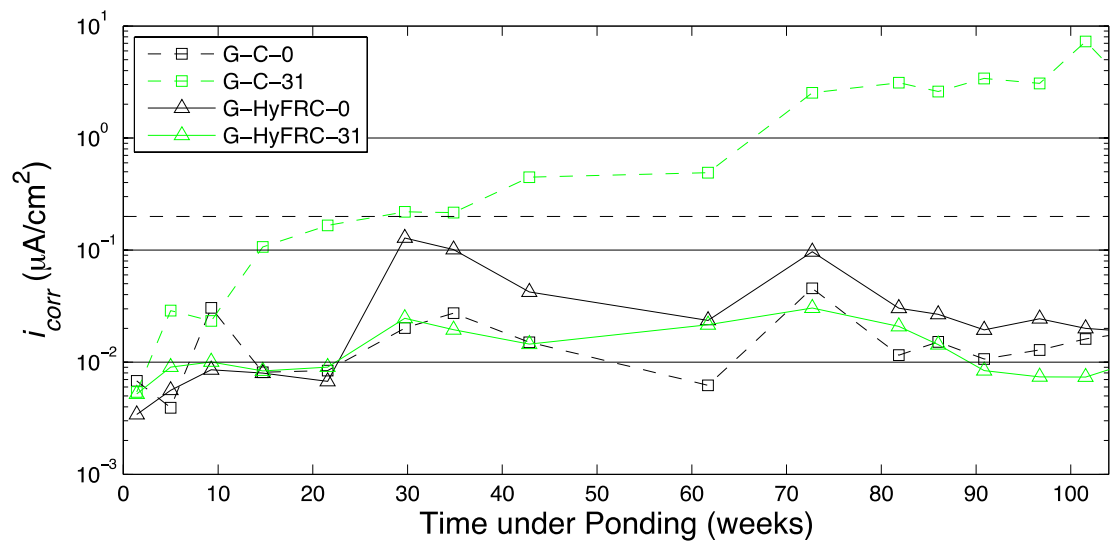

Figure 6. Microcell corrosion current density of No. 3 rebar in G-C and G-HyFRC beams.

Inclusion of fibers following the multi-scale hybridization approach was found to be effective for enhancing durability. When subjected to the same cyclic peak preload of $31 \mathrm{kN}$, green HyFRC composites were able to resist any observable corrosion-induced damage for up to two years, with corrosion current density being similar to green HyFRC specimens that were not preloaded. In the same time, green concrete composites without fiber reinforcement incurred significant splitting crack damage caused by corrosion damage (Figure 7). Compared to the initial damage caused by preloading, splitting cracks with considerable crack width propagated along the length of the beam. Similar splitting cracks were observed on the underside of the beam (horizontal surface nearest the No. 6 rebar), indicating the penetration of chlorides to the No. 6 rebar, a depth of $108 \mathrm{~mm}$ from the ponding surface. Concrete with severe splitting crack damage results in mechanical bond degradation between the concrete and rebar. In the condition when the differential slippage between the rebar and concrete is great, effective composite behavior between the concrete and rebar may be lost, leading to significant reduction in residual flexural capacity and premature replacement (Yoon et al., 2000). Based on the effective crack resistance of G-HyFRC, it is anticipated that the hybrid fiber reinforcement scheme described herein, when properly designed, could be adaptable to a range of concrete materials where emphasis on the origin of the batching material is prioritized for reducing total embodied energy of the resulting concrete.



(a)



(b)

Figure 7. Ponding surface of a G-C-31 beam: (a) After preloading, with flexural crack highlighted; (b) After 105 weeks of corrosion testing. 


\section{Corrosion behavior of HP-G-HyFRC cylinders}

Pertinent corrosion characteristics of the HP-G-C and HP-G-HyFRC specimens are presented in Table 4. The parameters were obtained at the conclusion of corrosion testing, which included the duration of impressed current (either $24 \mathrm{~h}$ or $48 \mathrm{~h}$ ) as well as the following 14-day bathing period, during which no impressed current was applied. It was assumed that any additional corrosion-induced damage during the 14-day period was minimal relative to the damage caused by accelerated testing. Values of $i_{\text {corr }}$ were averaged among readings taken at 1 day, 3 days, 7 days and 14 days during the secondary period. Mass loss of the rebar was obtained after splitting the specimens, extracting the bars, and cleaning the bars.

\section{Table 4. Corrosion characteristics after corrosion testing, including 14-day secondary bathing period.}

\begin{tabular}{|l|l|l|l|l|}
\hline & $\begin{array}{l}i_{\text {corr }} \\
\left(\mu \mathrm{A} / \mathrm{cm}^{2}\right)\end{array}$ & $\begin{array}{l}\text { Rebar } \\
\text { mass loss } \\
(\mathrm{g})\end{array}$ & $\begin{array}{l}\text { Percentage rebar mass } \\
\text { loss }(\%)\end{array}$ & $\begin{array}{l}\text { Estimated percentage rebar } \\
\text { mass loss from 24 } \mathrm{h}-48 \mathrm{~h} \\
(\%)\end{array}$ \\
\hline HP-G-C-24H & 4.11 & 4.1 & 0.76 & - \\
\hline HP-G-C-48H & 4.50 & 7.0 & 1.30 & 0.54 \\
\hline HP-G-HyFRC-24H & 1.49 & 3.2 & 0.59 & - \\
\hline HP-G-HyFRC-48H & 2.05 & 5.1 & 0.95 & 0.36 \\
\hline
\end{tabular}

As expected, higher measurements of current density were found for the longer duration of impressed current. However, for HP-G-C specimens, the change in $i_{\text {corr }}$ between $24 \mathrm{~h}$ and $48 \mathrm{~h}$ of accelerated corrosion testing was found to be small, increasing by $9.5 \%$ despite $100 \%$ increase in accelerated testing duration. HP-G-HyFRC exhibited lower current densities, being 36.3\% and 45.6\% of the HP-G-C specimens for 24 $\mathrm{h}$ and $48 \mathrm{~h}$ of testing, respectively. Correspondingly, the rebar mass loss was also found to be lower for HPG-HyFRC samples.

Figure 8 shows cross-sectional images of cut specimens after the conclusion of corrosion testing. To improve visibility, cracks were outlined with a black pen. Cracking in the HP-G-C matrix was characterized by a few, dominant cracks. These cracks would manifest as splitting cracks in structural elements, similar to the type previously observed in Figure 7. In contrast, addition of fiber hybridization resulted in more numerous, though finer, cracks. The large crack width in the HP-G-C specimens allowed for a greater ingress of sodium chloride solution and outward migration of corrosion products, resulting in greater corrosion activity. In contrast, as corrosion products formed and produced cracks in HP-G-HyFRC, fibers restricted crack propagation, initiating microcracks elsewhere in the matrix.

Due to this mechanical confinement, corrosion products collected at the steel-matrix interface, locally densifying there. This densification of the corrosion products at the interface is thought to reduce the oxidation rate of iron by the impressed current. When comparing the percentage of mass loss between the specimens, HP-G-HyFRC-24H lost $0.59 \%$ of its rebar mass for 24 total hours of impressed current, while HP-G-HyFRC-48H lost proportionally less rebar mass (approximately $0.36 \%$ ) from $24 \mathrm{~h}$ to $48 \mathrm{~h}$ despite increasing in $i_{\text {corr }}$, assuming similar corrosion behavior within the initial $24 \mathrm{~h}$ between the two HP-GHyFRC specimens. The nonlinear rebar mass loss was proposed by Liu and Weyers (1998), who suggested the rate of corrosion product growth decreased in proportion to the amount of product. HP-G-C, lacking confinement effects provided by hybrid fiber reinforcement, was not as effective in reducing the rebar mass loss from $24 \mathrm{~h}$ to $48 \mathrm{~h}$, losing approximately $0.54 \%$ of rebar mass compared to $0.36 \%$ lost in HP-G-HyFRC$48 \mathrm{H}$ during the same time period. 


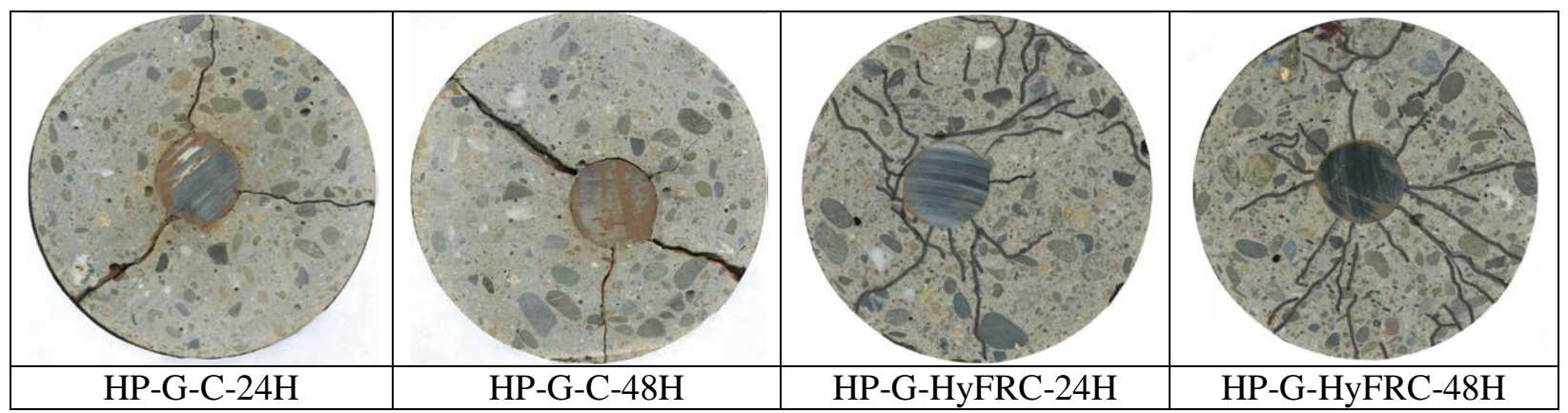

Figure 8. Cross sectional images of HP-G-C and HP-G-HyFRC after corrosion testing. Cracks are marked for improved visibility.

\section{CONCLUSION}

A series of experiments were performed to investigate the long-term durability enhancement of hybrid fiber-reinforced concrete (HyFRC) compared to conventional concrete. Use of HyFRC or HyFRC variants was found in all experiments to significantly reduce corrosion activity and related damage by providing mechanical control of cracks, despite corrosion being an electrochemical phenomenon. Such control is proposed as an effective method to increase the service life of structural composites, making these structures more sustainable by having greater longevity and not requiring extensive rehabilitation while in service. It is expected that a number of novel sustainable alternatives to conventional batching materials would be compatible for the durability enhancements provided by hybrid fiber reinforcement based on the satisfactory performance of the HyFRC variants described herein. In particular, the following conclusions can be made:

1. The flexural and splitting crack resistance provided by hybrid fiber reinforcement is beneficial for reinforced concrete structures subjected to crack-inducing service loads. Splitting cracks were found to be most detrimental to reducing time to corrosion initiation. Because hybrid fiber reinforcement suppressed splitting crack formation, as well as minimized flexural crack openings, HyFRC specimens loaded to $54 \mathrm{kN}$ behaved in a passive state up to 52 weeks, while plain concrete specimens at the same load level reached active corrosion after 12 weeks and had $i_{\text {corr }}$ measurements in excess of $1 \mu \mathrm{A} / \mathrm{cm}^{2}$.

2. To increase the proportion of recycled byproducts in concrete, the hybrid fiber reinforcement design was adopted for a high-volume fly ash (50\% mass replacement) matrix with coarse aggregates consisting entirely of recycled concrete. Preloaded reinforced beams cast with this green HyFRC (G-HyFRC) exhibited no active corrosion, based on $i_{c o r r}$ measurements, for 2 years. When cast without fiber reinforcement, preloaded green concrete beams exhibited active corrosion after only 14 weeks. Significant corrosion-induced splitting crack formed during the second year of experimentation, likely resulting in high loss of rebar-matrix mechanical bond.

3. A high-performance green HyFRC (HP-G-HyFRC) with 60\% replacement of portland cement with fly ash and slag was investigated under impressed current testing methods. Compared to samples without hybrid fiber reinforcement, HP-G-HyFRC formed numerous radial microcracks rather than a few, dominant macrocracks. The change in cracking characteristics caused by the fibers allowed corrosion products to densify at the rebar-matrix interface, decreasing corrosion activity and lowering rebar mass loss. 


\section{ACKNOWLEDGEMENT}

This work was supported in part by the Federal Highway Administration Grant DTFH61-11-C-00016 and in part by the Republic of Singapore's National Research Foundation though a grant to the Berkeley Alliance for Research in Singapore (BEARS) for the Singapore-Berkeley Building Efficiency and Sustainability in the Tropics (SinBerBEST) Program.

\section{REFERENCES}

American Society of Civil Engineers. (2013). 2013 Report card for America's infrastructure. <http://www.infrastructurereportcard.org>.

Andrade, C. and Alonso, C. (1996). "Corrosion rate monitoring in the laboratory and on-site." Constr Build Mater, 10(5), 315-328.

Berrocal, C.G., Löfgren, I., Lundgren, K. and Tang, L. (2015). "Corrosion initiation in cracked fibre reinforced concrete: Influence of crack width, fibre type and loading conditions." Corros Sci, 98, 128139.

Blunt, J. (2008). The effect of fiber reinforcement on the corrosion controlled degradation of reinforced concrete flexure elements. $\mathrm{PhD}$, University of California, Berkeley.

Blunt, J. and Ostertag, C.P. (2009). "Performance-based approach for the design of a deflection hardened hybrid fiber-reinforced concrete." J Eng Mech, 135(9), 978-986.

Ghods, P., Isgor, O.B., McRae, G.A., Li, J. and Gu, G.P. (2011). "Microscopic investigation of mill scale and its proposed effect on the variability of chloride-induced depassivation of carbon steel rebar." Corros Sci, 53(3), 946-954.

Goto, Y. (1971). "Cracks formed in concrete around deformed tension bars." ACI J Proc, 68(4), 244-251.

Hay, R. and Ostertag, C. (2014). "Development and application of high performance green hybrid fiberreinforced concrete (HP-G-HyFRC) for sustainable and energy-efficient buildings." Key Eng Mater, 629-630, 299-305.

IEA-WBCSD (2009). Cement technology roadmap 2009 - carbon emissions reductions up to 2050.

Koch, G.H., Brongers, M.P.H., Thompson, N.G., Virmani, Y.P. and Payer, J.H. (2002). Corrosion costs and preventive strategies in the United States. McLean, VA: Turner-Fairbank Highway Research Center.

Liu, Y. and Weyers, R.E. (1998). "Modeling the time-to-corrosion cracking in chloride contaminated reinforced concrete structures." ACI Mater J, 95(6), 675-681.

Miyazato, S. and Hiraishi, Y. (2013). "Durability against steel corrosion of HPFRCC with bending cracks." $J$ Adv Concr Techol, 11(4), 135-143.

Sahmaran, M., Li, V.C. and Andrade, C. (2008). "Corrosion resistance performance of steel-reinforced engineered cementitious composite beams." ACI Mater J, 105(3), 243-250.

Sahmaran, M. and Yaman, I.O. (2008). "Influence of transverse crack width on reinforcement corrosion initiation and propagation in mortar beams." Can J Civ Eng, 35(3), 236-245.

U.S. Geological Survey (2013). Mineral commodity summaries 2013. Reston, VA: U.S. Geological Survey.

Wang, K., Jansen, D.C., Shah, S.P. and Karr, A.F. (1997). "Permeability study of cracked concrete." Cem Concr Compos, 27(3), 381-393.

Yoon, S., Wang, K., Weiss, W.J. and Shah, S.P. (2000). "Interaction between loading, corrosion, and serviceability of reinforced concrete." ACI Mater J, 97(6), 637-644. 